\title{
DELTA-SHOCK WAVES AS SELF-SIMILAR VISCOSITY LIMITS
}

BY

\author{
GREY ERCOLE
}

\begin{abstract}
Departamento de Matemática, Universidade Federal de Minas Gerais, Caixa Postal 702, 30.123-970
\end{abstract} Belo Horizonte, Brazil

1. Introduction. We consider the Riemann problem for a system of conservation laws:

$$
\begin{gathered}
U_{t}+F(U)_{x}=0 ; \quad(x, t) \in \mathbf{R}_{+}^{2}, \\
U(x, 0)= \begin{cases}U_{l} ; & x<0, \\
U_{r} ; & x>0 .\end{cases}
\end{gathered}
$$

It is natural to search for weak solutions for (1.1)-(1.2) that are self-similar, which in this context means that they are invariant under dilations of the independent variables of the form $(x, t) \mapsto(a x, a t), a>0$, or in other words, that they are constant along the rays $x=a t, t>0$.

One of the standard methods for the study of the Riemann problem (1.1)-(1.2) is the vanishing viscosity method, which consists of introducing a term of the form $\varepsilon U_{x x}$ on the right-hand side of (1.1), which results in a parabolic system whose solutions depend on the parameter $\varepsilon$. One then proceeds to determine the behavior of the smooth solutions of this parabolic system when $\varepsilon \rightarrow 0^{+}$, with initial data (1.2) or some suitable regularization of it. One hopes to obtain existence and additional properties of the weak solutions of (1.1)-(1.2) this way. The vanishing viscosity regularization is often physically appropriate and, to an extent, preserves the Galilean invariance associated to the system (1.1) by approximating shock waves by travelling wave solutions of the regularized system. However, the self-similarity of the approximating solutions is lost.

If one wishes to preserve the self-similarity at the level of approximations, it is possible to employ a variant of the vanishing viscosity method that consists of introducing a term of the form $\varepsilon t U_{x x}$, on the right-hand side of (1.1). This gives rise to the regularized system:

$$
U_{t}+F(U)_{x}=\varepsilon t U_{x x} ; \quad(x, t) \in \mathbf{R}_{+}^{2}, \varepsilon>0 .
$$

Received April 28, 1998.

2000 Mathematics Subject Classification. Primary 35L65, 35L67.

E-mail address: grey@mat.ufmg.br 
This system often admits solutions that are smooth and self-similar, that satisfy (1.2) and which are called viscosity wavefans. These solutions can be written in the form $U_{\varepsilon}=U_{\varepsilon}(\xi)$, with $\xi=x / t$ and $U_{\varepsilon}$ satisfying the boundary value problem:

$$
\begin{gathered}
\varepsilon \ddot{U}=\dot{F}(u)-\xi \dot{U} ; \quad \xi \in(-\infty,+\infty), \\
U(-\infty)=U_{l}, \quad U(+\infty)=U_{r} .
\end{gathered}
$$

(The dot denotes $\frac{d}{d \xi}$.)

This approach was developed by C. Dafermos in [1]. Dafermos proved the existence of the viscosity wavefans $U_{\varepsilon}(\xi)$ using the Leray-Schauder fixed point theorem. Furthermore, he used Helly's lemma to prove the weak convergence of the viscosity wavefans under the hypothesis of uniform boundedness in $L^{\infty}$ and in total variation of the family $\left\{U_{\varepsilon}\right\}$. The existence result remains the best available but it requires an a priori estimate in $L^{\infty}$ which can be problematic to obtain. The application to $2 \times 2$ systems was developed by C. Dafermos and R. DiPerna in [2].

The theory of viscosity wavefans, as developed by Dafermos, has been applied to several examples of $2 \times 2$ systems, as in $[7,8,11,14]$, including systems that change type, $[3,4,10]$. Recently (see [15]), A. Tzavaras has employed the method of Dafermos, plus a careful local study of the behavior of the total variation of the solutions of (1.4)(1.5) to obtain convergence of the viscosity wavefans for Riemann problems with small amplitude.

In the present work we introduce a general framework for obtaining weak convergence of the viscosity wavefans which relies basically on $L^{1}$ uniform bounds on the viscosity wavefans, plus some additional pointwise information. We apply this framework to the following class of strictly hyperbolic Riemann problems:

$$
\begin{aligned}
& \left\{\begin{aligned}
& u_{t}+f(u)_{x}=0,(x, t) \in \mathbf{R}_{+}^{2}, \\
& v_{t}+(v g(u))_{x}=0,
\end{aligned}\right. \\
& (u(x, 0), v(x, 0))= \begin{cases}\left(u_{l}, v_{l}\right) ; & x<0, \\
\left(u_{r}, v_{r}\right) ; & x>0,\end{cases}
\end{aligned}
$$

where $f$ and $g$ are smooth functions depending only on the variable $u$ and satisfying

$$
g^{\prime}>0, \quad f^{\prime \prime}>0, \quad f^{\prime}<g .
$$

The weak solutions of (1.6) may involve superpositions of a shock wave with a Dirac mass, occurring at the same place. This kind of wave, called a delta-shock wave, was introduced by D. J. Korchinski in [9] and further studied by D. Tan, T. Zhang, and Y. Zheng in [13] for the Riemann problem with $f(u)=u^{2}$ and $g(u)=u$. This nonstrictly hyperbolic problem has no standard weak solutions (composed by standard shock waves, rarefaction waves, and contact discontinuities) for certain values of initial data $\left(u_{l}, v_{l}\right)$ and $\left(u_{r}, v_{r}\right)$, but the Riemann problem can be solved uniquely if one adds delta-shock waves with a suitable entropy condition to the list of allowed simple waves.

Also in [13], D. Tan, T. Zhang, and Y. Zheng stated that the Riemann problem (1.6)-(1.7) under the conditions (1.8) has no standard weak solutions when the initial data evolves to an overcompressive discontinuity. Furthermore, they claimed that the 
appropriate weak solution for this case is formed by one delta-shock wave. This fact is proved here, as an application of our $L^{1}$ framework for viscosity wavefans.

With respect to the specific strictly hyperbolic system (1.6) with the conditions (1.8) we note that a general result of well-posedness for the Cauchy problem was recently proved by F. Huang in [6]. He built approximate solutions for the first equation in (1.6) of both sides of the possible points of discontinuities by mollification of the Oleinik solution for this equation. Then, by making a careful study of the characteristic curves of a smoothing out of the second equation, he found the solution of the Cauchy problem as a weak limit. This solution is obtained in a generalized sense that employs the Lebesgue-Stieltjes integral specific for the system. Huang's entropy criterion agrees with the entropy criteria found here and in the literature for the specific case of Riemann data.

Huang's work gives a very satisfactory account for the theory of weak solutions to system (1.6) subject to (1.8). In this sense, the current work may, at best, be regarded as a structure theorem, studying in detail certain waves that appear in Huang's weak solutions. However, the $L^{1}$ framework for the convergence of viscosity wavefans developed here, although initially motivated by the Riemann problem for system (1.6), is of independent interest.

Our analysis begins by proving (just assuming $g^{\prime}>0$ ) existence and uniqueness of the viscosity wavefan $U_{\varepsilon}(x / t)=\left(u_{\varepsilon}(x / t), v_{\varepsilon}(x / t)\right)$, which is the solution of

$$
\left\{\begin{aligned}
u_{t}+f(u)_{x} & =\varepsilon t u_{x x} \\
v_{t}+(v g(u))_{x} & =\varepsilon t v_{x x}
\end{aligned}\right.
$$

satisfying (1.7). The uniqueness of the viscosity wavefans is a new observation, even for a single equation.

Our main result concerning systems of the form (1.9) is that, under the conditions (1.8) on $f$ and $g$, the viscosity wavefans $U_{\varepsilon}=\left(u_{\varepsilon}, v_{\varepsilon}\right)$ converge weakly to a Riemann solution of (1.6) composed of conventional simple waves and delta-shocks as described above. We call attention to the fact that such Riemann solutions are excluded from the original Dafermos framework, since no bounds in $L^{\infty}$ or in total variation can be expected to hold uniformly in the sequence. The best that one may expect is a uniform $L_{\text {loc }}^{1}$ bound.

The existence and convergence results for the viscosity wavefans were proved in [12] by D. Tan for the particular system of the form (1.9) studied in [13]. His proof of existence of the viscosity wavefans does not generalize to the class of systems under consideration, because he used the fact that each solution of the first equation in (1.9) is also a solution of the second (when $f(u)=u^{2}$ and $g(u)=u$ ). In addition, in view of the uniqueness that we have proved, the viscosity wavefans obtained in [12] coincide with the ones obtained here.

In [5], J. Hu extended the existence and convergence results of D. Tan. His approach departs sharply from what was done in [12], by adapting the general framework of DiPerna and Dafermos to this problem instead of relying on the rather special algebraic properties of the solution of (1.9). In this sense, [5] is very close in spirit to the present work, and a substantial part of our work may be regarded as an independently obtained extension of the work in [5]. Aside from the fact that [5] and the present work 
deal with different systems, there are two aspects in which this work goes beyond [5]. One is the general framework developed in Sec. 2 for an $L^{1}$ theory of convergence of viscosity wavefans, of which the work in [5] includes a very particular instance. The second aspect is that in the proof of Theorem 4.3 (i) and (ii) in [5], J. Hu used the RankineHugoniot condition to obtain the convergence of the viscosity wavefans in the case where the Riemann solution is composed of a classical shock and a contact discontinuity. There is no reason for a weak limit that is not a weak solution to satisfy the Rankine-Hugoniot condition. The technique for bypassing the difficulty here is based on an observation by B. Keyfitz and H. Kranzer [8] (Theorem 2.3 in this paper), and which is part of the general $L^{1}$ framework that is the main purpose of this work.

The paper is organized as follows:

In Sec. 2 we present the general results about the viscosity wavefans $U_{\varepsilon}$ associated to $n \times n$ Riemann problems (1.1)-(1.2) that constitute the $L^{1}$ framework mentioned above. The main results are the weak convergence of $U_{\varepsilon}$ and $F\left(U_{\varepsilon}\right)$, as $\varepsilon \rightarrow 0^{+}$, for distributions involving Dirac delta functions, assuming that the viscosity wavefans are uniformly bounded in $L_{\text {loc }}^{1}$ and satisfy certain pointwise estimates away from the shocks.

In Sec. 3 we present the global weak solution of the Riemann problem (1.6)-(1.7) under the conditions (1.8). This follows the closely related work [13].

In Sec. 4 we prove existence, uniqueness and a priori $L^{\infty}$ and $L_{\text {loc }}^{1}$-estimates of the viscosity wavefans associated with (1.6)-(1.7). For this purpose we make a study of critical points. All results of this section can be proved for a system similar to (1.6) with the second equation replaced by a more general equation that is nonlinear in $v$ (see Remark 4.1).

Finally, in Sec. 5 we prove the weak convergence of the viscosity wavefans associated with (1.6)-(1.7). We consider only the case $u_{l}>u_{r}$ in which the solution of (1.6)(1.7) is composed of one shock wave or one delta-shock wave followed by one contact discontinuity. Since our principal interest is the weak convergence for delta-shock waves, we give the proof in detail for this case and indicate the crucial points in the proof of the weak convergence for the classical shock waves. We observe that the $L^{\infty}$-estimate obtained in Sec. 4 is $O(1 / \varepsilon)$ which is sufficient to prove existence but not to prove convergence by arguments based on Helly's lemma.

In the case $u_{l} \leq u_{r}$ the weak solution is composed of one rarefaction wave followed by one contact discontinuity and the weak convergence can be obtained via the Dafermos framework.

2. Viscosity wavefans. In this section we consider the general viscosity wavefans of order $n$, i.e., the smooth solutions $U_{\varepsilon}$ of the boundary value problem (1.4)-(1.5) with a smooth function $F: \mathbf{R}^{n} \rightarrow \mathbf{R}^{n}$.

We present some results that will be used in this work and prove that, under some conditions, $U_{\varepsilon}$ converges weakly to a distribution $D$ involving Dirac delta functions. This distribution is a weak solution of (1.4)-(1.5) providing that $F(D)$ is defined in an appropriate manner. Moreover, we prove that, under an additional condition, $F\left(U_{\varepsilon}\right)$ converges to $F(D)$. 
We begin by stating two estimates on the first derivative of the viscosity wavefans which can be found in [15].

Proposition 2.1. Let $U_{\varepsilon}=U_{\varepsilon}(\xi)$ be a smooth solution of (1.4)-(1.5). Then

$$
\left\|\dot{U}_{\varepsilon}(\xi)\right\| \leq\left\|\dot{U}_{\varepsilon}(0)\right\| \exp \left(\frac{2 \alpha_{\varepsilon}|\xi|-\xi^{2}}{2 \varepsilon}\right) ; \quad \xi \in \mathbf{R}
$$

and

$$
\left\|\dot{U}_{\varepsilon}(\xi)\right\| \leq \frac{C}{\varepsilon}\left[\beta_{\varepsilon}+(1+|\xi|)\left\|U_{\varepsilon}\right\|_{\infty}\right] ; \quad \xi \in \mathbf{R},
$$

where $\alpha_{\varepsilon}=\sup \left\{\left\|d F\left(U_{\varepsilon}(\xi)\right)\right\|: \xi \in \mathbf{R}\right\}, \beta_{\varepsilon}=\sup \left\{\left\|F\left(U_{\varepsilon}(\xi)\right)\right\|: \xi \in \mathbf{R}\right\}$ and $C$ is a constant that does not depend on $\varepsilon$.

REMARK 2.1. The estimate (2.1) shows that the viscosity wavefans decay rapidly to zero when $|\xi| \rightarrow+\infty$, for each fixed $\varepsilon>0$. On the other hand, the estimate (2.2) can be used on a closed interval $I$ to obtain estimates of the form $\left\|\dot{U}_{\varepsilon}\right\|_{L^{\infty}(I)}=O\left(1 / \varepsilon^{m}\right)$. For example, if $I=[a, b]$ then from $(2.2)$ it follows that

$$
\left\|\dot{U}_{\varepsilon}(\xi)\right\| \leq \frac{K_{I}}{\varepsilon}\left(\beta_{\varepsilon}+\left\|U_{\varepsilon}\right\|_{\infty}\right) ; \quad \xi \in I,
$$

where $K_{I}=C(1+\max \{|b|,|a|\})$ is a constant that depends only on $I$. Thus, if $\beta_{\varepsilon}=$ $O\left(1 / \varepsilon^{p}\right)$ and $\left\|U_{\varepsilon}\right\|_{\infty}=O\left(1 / \varepsilon^{p}\right)$, then $\left\|\dot{U}_{\varepsilon}\right\|_{L^{\infty}(I)}=O\left(1 / \varepsilon^{p+1}\right)$ on $I$.

Next we state, without proof, a Theorem due to Dafermos [1] which is the only general result known about the existence of a smooth solution for (1.4)-(1.5). Its proof is based on the Leray-Schauder fixed-point theorem.

Theorem 2.2. Consider the following boundary value problem with parameters $\mu \in$ $[0,1]$ and $L \geq 1$ :

$$
\left\{\begin{array}{l}
\varepsilon \ddot{U}=\mu \dot{F}(U)-\xi \dot{U} ; \quad \xi \in(-L,+L), \\
U(-L)=\mu U_{l}, \quad U(L)=\mu U_{r}
\end{array}\right.
$$

Suppose there exists a constant $M$, depending at most on $\varepsilon, U_{l}$, and $U_{r}$ (and thus independent of $\mu$ and $L$ ) such that

$$
\sup _{\xi \in(-L, L)}\|U(\xi)\| \leq M
$$

for all smooth solutions $U(\xi)$ of $(2.3)$.

Then there exists a smooth solution (not necessarily unique) of (1.4)-(1.5) satisfying (2.4) with $L=\infty$.

The following result, due to Keyfitz and Kranzer [8], provides an interesting relation, which is independent of $\varepsilon$, between the viscosity wavefans and the discontinuous functions of the form

$$
W_{s}(\xi)= \begin{cases}U_{l} ; & \xi<s \\ U_{r} ; & \xi>s\end{cases}
$$


THEOREM 2.3. Let $U_{\varepsilon}=U_{\varepsilon}(\xi)$ be a smooth solution of (1.4)-(1.5). Then, for each $s \in \mathbf{R}$, we have $\int_{-\infty}^{+\infty}\left[U_{\varepsilon}(\xi)-W_{s}(\xi)\right] d \xi=s\left[U_{r}-U_{l}\right]-\left[F\left(U_{r}\right)-F\left(U_{l}\right)\right]$, where $W_{s}$ is defined by (2.5).

Now we prove the main results of this section which show that, under certain conditions, $U_{\varepsilon} \rightarrow D$ and $F\left(U_{\varepsilon}\right) \rightarrow F(D)$ when $\varepsilon \rightarrow 0^{+}$. Here $D$ and $F(D)$ are the distributions defined respectively by

$$
D=V+\sum_{j=1}^{k} C_{j} \delta_{\sigma_{j}}
$$

and

$$
F(D)=F(V)+\sum_{j=1}^{k} \sigma_{j} C_{j} \delta_{\sigma_{j}}
$$

where $\sigma_{0}=-\infty<\sigma_{1}<\cdots<\sigma_{k}<\sigma_{k+1}=\infty, V=V(\xi)$ is a piecewise continuous function that is a classical solution of the system in (1.4) on each interval $I_{j}=\left(\sigma_{j}, \sigma_{j+1}\right), \delta_{\sigma_{j}}$ is the Dirac $\delta$-function concentrated at $\sigma_{j}$ and

$$
C_{j}=\sigma_{j}\left[V\left(\sigma_{j}^{+}\right)-V\left(\sigma_{j}^{-}\right)\right]-\left[F\left(V\left(\sigma_{j}^{+}\right)\right)-F\left(V\left(\sigma_{j}^{-}\right)\right)\right] .
$$

THEOREM 2.4. For each $\varepsilon>0$, let $U_{\varepsilon}=U_{\varepsilon}(\xi)$ be a smooth function of (1.4)-(1.5). Suppose that

(i) $U_{\varepsilon}$ converges uniformly to a function $V$ as above on closed intervals contained in $I_{j}=\left(\sigma_{j}, \sigma_{j+1}\right)$ for all $j \in\{0,1,2, \ldots, k\}$;

(ii) the family $\left\{U_{\varepsilon}\right\}$ is uniformly bounded in $L_{\text {loc }}^{1}(\mathbf{R})$, i.e., for each closed interval $I \subset \mathbf{R}$, there exists a constant $M$ (which can depend on $I$, but not on $\varepsilon$ ) such that $\int_{I}\left\|U_{\varepsilon}\right\| d \xi \leq M$ for all $\varepsilon>0$.

Then $U_{\varepsilon} \rightarrow D$, where $D$ is the distribution defined by (2.6).

Proof. We must show that

$$
\lim _{\varepsilon \rightarrow 0^{+}} \int_{-\infty}^{+\infty}\left[U_{\varepsilon}-V\right] \phi d \xi=\sum_{j=1}^{k} C_{j} \phi\left(\sigma_{j}\right) ; \quad \phi \in C_{0}^{\infty}(\mathbf{R}) .
$$

Initially we prove (2.9) for $\phi \in C_{0}^{\infty}(\mathbf{R})$ such that supp $\phi \subset\left[\sigma_{j}-\alpha, \sigma_{j}+\alpha\right]$ for fixed $j \in\{1,2, \ldots, k\}$ with $\sigma_{i} \notin\left[\sigma_{j}-\alpha, \sigma_{j}+\alpha\right]$ if $i \neq j$ for some $\alpha>0$. Thus, the equality in (2.9) reduces to

$$
\lim _{\varepsilon \rightarrow 0^{+}} \int_{-\infty}^{+\infty}\left(U_{\varepsilon}-V\right) \phi d \xi=C_{j} \phi\left(\sigma_{j}\right) .
$$

Following [13], we fix $\phi$ as above and take $\psi \in C_{0}^{\infty}(\mathbf{R})$ such that

$$
\psi(\xi) \equiv \phi\left(\sigma_{j}\right) ; \quad \xi \in\left[\sigma_{j}-\beta, \sigma_{j}+\beta\right] \subset\left(\sigma_{j}-\alpha, \sigma_{j}+\alpha\right),
$$

$\operatorname{supp} \psi \subset\left[\sigma_{j}-\alpha, \sigma_{j}+\alpha\right]$ and $\|\psi-\phi\|_{\infty} \leq \tau$, where $\tau$ is a small positive constant. The function $\psi$ is a sloping test function near to $\phi$. 
Multiplying (1.4) by $\psi$ and integrating by parts on $(-\infty,+\infty)$ we obtain

$$
\int_{-\infty}^{+\infty} U_{\varepsilon} \psi d \xi=I_{1}^{\varepsilon}-I_{2}^{\varepsilon}
$$

where $I_{1}^{\varepsilon}=\int_{-\infty}^{+\infty}\left[F\left(U_{\varepsilon}\right)-\xi U_{\varepsilon}\right] \dot{\psi} d \xi$ and $I_{2}^{\varepsilon}=\varepsilon \int_{-\infty}^{+\infty} \dot{U}_{\varepsilon} \dot{\psi} d \xi=-\varepsilon \int_{-\infty}^{+\infty} U_{\varepsilon} \ddot{\psi} d \xi$.

We have $\lim _{\varepsilon \rightarrow 0^{+}} I_{2}^{\varepsilon}=0$ since $\left\|I_{2}^{\varepsilon}\right\| \leq \varepsilon\|\ddot{\psi}\|_{\infty} \int_{\sigma_{j}-\alpha}^{\sigma_{j}+\alpha}\left\|U_{\varepsilon}\right\| d \xi$ and $U_{\varepsilon}$ is uniformly bounded in $L_{\text {loc }}^{1}(\mathbf{R})$.

From hypothesis (i) we have

$$
\begin{aligned}
\lim _{\varepsilon \rightarrow 0^{+}} I_{1}^{\varepsilon} & =\lim _{\varepsilon \rightarrow 0^{+}}\left(\int_{\sigma_{j}-\alpha}^{\sigma_{j}-\beta}+\int_{\sigma_{j}+\beta}^{\sigma_{j}+\alpha}\right)\left(F\left(U_{\varepsilon}\right)-\xi U_{\varepsilon}\right) \dot{\psi} d \xi \\
& =\left(\int_{\sigma_{j}-\alpha}^{\sigma_{j}-\beta}+\int_{\sigma_{j}+\beta}^{\sigma_{j}+\alpha}\right)(F(V)-\xi V) \dot{\psi} d \xi=\int_{\sigma_{j}-\alpha}^{\sigma_{j}+\alpha}(F(V)-\xi V) \dot{\psi} d \xi .
\end{aligned}
$$

Using integration by parts on $\left[\sigma_{j}-\alpha, \sigma_{j}\right) \cup\left(\sigma_{j}, \sigma_{j}+\alpha\right]$ and noting that $\psi\left(\sigma_{j}-\alpha\right)=$ $\psi\left(\sigma_{j}+\alpha\right)=0$ and $\psi\left(\sigma_{j}\right)=\phi\left(\sigma_{j}\right)$ we obtain

$$
\begin{aligned}
\lim _{\varepsilon \rightarrow 0^{+}} I_{1}^{\varepsilon} & =\phi\left(\sigma_{j}\right)[F(V(\xi))-\xi V(\xi)]_{\xi=\sigma_{j}^{+}}^{\xi=\sigma_{j}^{-}}+\int_{\sigma_{j}-\alpha}^{\sigma_{j}+\alpha} V \psi d \xi \\
& =C_{j} \phi\left(\sigma_{j}\right)+\int_{-\infty}^{+\infty} V \psi d \xi .
\end{aligned}
$$

Therefore, in view of (2.12) we conclude that

$$
\lim _{\varepsilon \rightarrow 0^{+}} \int_{-\infty}^{+\infty}\left(U_{\varepsilon}-V\right) \psi d \xi=C_{j} \phi\left(\sigma_{j}\right)
$$

Now, from hypothesis (ii) it follows that

$$
\int_{\sigma_{j}-\alpha}^{\sigma_{j}+\alpha}\left\|U_{\varepsilon}-V\right\| d \xi \leq L
$$

for some positive constant $L$ that is independent of $\varepsilon>0$. So,

$$
\begin{aligned}
& \left\|\int_{-\infty}^{+\infty}\left(U_{\varepsilon}-V\right) \phi d \xi-C_{j} \phi\left(\sigma_{j}\right)\right\| \\
& \quad \leq\|\phi-\psi\|_{\infty} \int_{\sigma_{j}-\alpha}^{\sigma_{j}+\alpha}\left\|U_{\varepsilon}-V\right\| d \xi+\left\|\int_{\sigma_{j}-\alpha}^{\sigma_{j}+\alpha}\left(U_{\varepsilon}-V\right) \psi d \xi-C_{j} \phi\left(\sigma_{j}\right)\right\| \\
& \quad \leq \tau L+\left\|\int_{\sigma_{j}-\alpha}^{\sigma_{j}+\alpha}\left(U_{\varepsilon}-V\right) \psi d \xi-C_{j} \phi\left(\sigma_{j}\right)\right\| .
\end{aligned}
$$

In view of (2.13) we have $\lim _{\varepsilon \rightarrow 0^{+}}\left\|\int_{-\infty}^{+\infty}\left(U_{\varepsilon}-V\right) \phi d \xi-C_{j} \phi\left(\sigma_{j}\right)\right\| \leq \tau L$ which leads to (2.10) when $\tau \rightarrow 0^{+}$.

If $\phi \in C_{0}^{\infty}(\mathbf{R})$ is arbitrary, then we take $\phi_{1}, \phi_{2}, \ldots, \phi_{k} \in C_{0}^{\infty}(\mathbf{R})$ such that $\sigma_{i} \notin \operatorname{supp} \phi_{j}$ if $i \neq j$ and

$$
\sum_{i=1}^{k} \phi_{i}=\phi
$$


Thus, we obtain (2.9) from (2.10) applied to each $\phi_{i}$.

Under an additional hypothesis to the last theorem we obtain the weak convergence of $F\left(U_{\varepsilon}\right)$.

Theorem 2.5. For each $\varepsilon>0$ let $U_{\varepsilon}$ be a solution of (1.4)-(1.5). Suppose (i)-(ii) of Theorem 2.4 and the additional hypothesis:

(iii) for each $\xi \in \mathbf{R}-\left\{\sigma_{1}, \sigma_{2}, \ldots, \sigma_{k}\right\}$ the sequence $\left\{\dot{U}_{\varepsilon}(\xi)\right\}$ is bounded.

Then, $F\left(U_{\varepsilon}\right) \rightarrow F(D)$, where $F(D)$ is the distribution defined by $(2.7)$.

Proof. Let $j \in\{1,2, \ldots, k\}$ be fixed and let $\varphi \in C_{0}^{\infty}(\mathbf{R})$ be a test function with $\operatorname{supp} \varphi \subset I=[a, b], \sigma_{j} \in I$ and $\sigma_{i} \notin I$ if $i \neq j$.

Integrating (1.4) by parts we obtain

$$
F\left(U_{\varepsilon}(\xi)\right)=\left[F\left(U_{\bar{\varepsilon}}\right)-\varepsilon \dot{U}_{\varepsilon}-a U_{\varepsilon}\right]_{\xi=a}+\varepsilon \dot{U}_{\varepsilon}(\xi)+\xi U_{\varepsilon}(\xi)-\int_{a}^{\xi} U_{\varepsilon}(\eta) d \eta .
$$

Therefore,

$$
\int_{-\infty}^{+\infty} F\left(U_{\varepsilon}\right) \varphi d \xi=I_{\varepsilon}^{1}+I_{\varepsilon}^{2}+I_{\varepsilon}^{3}-I_{\varepsilon}^{4}
$$

where $I_{\varepsilon}^{1}=\left[F\left(U_{\varepsilon}\right)-\varepsilon \dot{U}_{\varepsilon}-\xi U_{\varepsilon}\right]_{\xi=a} \int_{a}^{b} \varphi d \xi, I_{\varepsilon}^{2}=\varepsilon \int_{a}^{b} \dot{U}_{\varepsilon} \varphi d \xi=-\varepsilon \int_{a}^{b} U_{\varepsilon} \dot{\varphi} d \xi, I_{\varepsilon}^{3}=$ $\int_{a}^{b} \xi U_{\varepsilon} \varphi d \xi$, and $I_{\varepsilon}^{4}=\int_{a}^{b} \varphi \int_{a}^{\xi} U_{\varepsilon} d \eta d \xi$.

From hypothesis (ii) we have immediately $\lim _{\varepsilon \rightarrow 0^{+}} I_{\varepsilon}^{2}=0$ and from hypotheses (i) and (iii) it follows that $\lim _{\varepsilon \rightarrow 0^{+}} I_{\varepsilon}^{1}=[F(V)-\xi V]_{\xi=a} \int_{a}^{b} \varphi d \xi$.

Since $U_{\varepsilon} \rightarrow D$ (Theorem 2.4) we have $\lim _{\varepsilon \rightarrow 0^{+}} I_{\varepsilon}^{3}=\int_{a}^{b} \xi V \varphi d \xi+C_{j} \sigma_{j} \varphi\left(\sigma_{j}\right)$.

Now, from hypothesis (ii), we obtain

$$
\left\|\varphi(\xi) \int_{a}^{\xi} U_{\varepsilon}(\eta) d \eta\right\| \leq\|\varphi\|_{\infty} \int_{a}^{b}\left\|U_{\varepsilon}(\eta)\right\| d \eta \leq L ; \quad \xi \in I,
$$

for some $L>0$ that is independent of $\varepsilon$. So,

$$
\lim _{\varepsilon \rightarrow()^{+}} I_{\varepsilon}^{4}=\int_{a}^{b} \varphi(\xi)\left[\lim _{\varepsilon \rightarrow 0^{+}} \int_{a}^{\xi} U_{\varepsilon}(\eta) d \eta\right] d \xi .
$$

In order to simplify (2.17) we calculate the function

$$
\lim _{\varepsilon \rightarrow 0^{+}} \int_{a}^{\xi} U_{\varepsilon}(\eta) d \eta ; \quad \text { a.e. } \xi \in[a, b] .
$$

Let $\xi \in[a, b]$ be fixed. If $a<\xi<\sigma_{j}$ then, in view of the (uniform) convergence $U_{\varepsilon} \rightarrow V$ on $[a, \xi]$ and of the equality $F(V(\eta))=\eta \dot{V}(\eta), \eta \in\left[a, \sigma_{j}\right)$, we have

$$
\lim _{\varepsilon \rightarrow 0^{+}} \int_{a}^{\xi} U_{\varepsilon}(\eta) d \eta=\int_{a}^{\xi} V(\eta) d \eta=-[F(V(\eta))-\eta V(\eta)]_{a}^{\xi} .
$$

On the other hand, if $\xi>\sigma_{j}$, let $\alpha>0$ be such that $a+\alpha<\sigma_{j}<\xi-\alpha$ and let $\psi \in C_{0}^{\infty}(\mathbf{R})$ be a test function satisfying $\operatorname{supp} \psi \subset(a, \xi)$ and $\psi(\eta)=1$ if $\eta \in[a+\alpha, \xi-\alpha]$. 
Then

$$
\begin{aligned}
\int_{a}^{\xi}\left(U_{\varepsilon}-V\right) d \eta & =\int_{a}^{\xi}\left(U_{\varepsilon}-V\right)(1-\psi) d \eta+\int_{a}^{\xi}\left(U_{\varepsilon}-V\right) \psi d \eta \\
& =\left(\int_{a}^{a+\alpha}+\int_{\xi-\alpha}^{\xi}\right)\left(U_{\varepsilon}-V\right)(1-\psi) d \eta+\int_{-\infty}^{+\infty}\left(U_{\varepsilon}-V\right) \psi d \eta
\end{aligned}
$$

Since $U_{\varepsilon} \rightarrow V$ uniformly on $[a, a+\alpha]$ we have

$$
\lim _{\varepsilon \rightarrow 0^{+}}\left\|\int_{a}^{a+\alpha}\left(U_{\varepsilon}-V\right)(1-\psi) d \eta\right\| \leq\|1-\psi\|_{\infty} \lim _{\varepsilon \rightarrow 0^{+}} \int_{a}^{a+\alpha}\left\|U_{\varepsilon}-V\right\| d \eta=0 .
$$

Similarly, we obtain $\lim _{\varepsilon \rightarrow 0^{+}}\left\|\int_{\xi-\alpha}^{\xi}\left(U_{\varepsilon}-V\right)(1-\psi) d \eta\right\|=0$.

So, $\lim _{\varepsilon \rightarrow 0^{+}} \int_{a}^{\xi}\left[U_{\varepsilon}-V\right] d \eta=\lim _{\varepsilon \rightarrow 0^{+}} \int_{-\infty}^{+\infty}\left(U_{\varepsilon}-V\right) \psi d \eta=C_{j} \psi\left(\sigma_{j}\right)=C_{j}$, if $\xi \in\left(\sigma_{j}, b\right]$. (We have used Theorem 2.4 to obtain the second equality.)

Therefore, integrating by parts the equality $\dot{F}(V(\eta))=\eta \dot{V}(\eta)$ satisfied for $\eta \in\left(a, \sigma_{j}\right) \cup$ $\left(\sigma_{j}, b\right]$, we can verify that $\int_{a}^{\xi} V(\eta) d \eta+C_{j}=-[F(V(\eta))-\eta V(\eta)]_{a}^{\xi}$, if $\xi \in\left(\sigma_{j}, b\right]$.

We have concluded that $\lim _{\varepsilon \rightarrow 0^{+}} \int_{a}^{\xi} U_{\varepsilon}(\eta) d \eta=-[F(V(\eta))-\eta V(\eta)]_{a}^{\xi}$, for all $\xi \in$ $\left[a, \sigma_{j}\right) \cup\left(\sigma_{j}, b\right]$ and hence, $\lim _{\varepsilon \rightarrow 0^{+}} I_{\varepsilon}^{4}=-\int_{a}^{b} \varphi(\xi)[F(V(\eta))-\eta V(\eta)]_{a}^{\xi} d \xi$.

Now, from (2.16) we have $\lim _{\varepsilon \rightarrow 0^{+}} \int_{a}^{b} F\left(U_{\varepsilon}\right) \varphi d \xi=\int_{a}^{b} F(V) \varphi d \xi+C_{j} \sigma_{j} \varphi\left(\sigma_{j}\right)$, i.e.,

$$
\lim _{\varepsilon \rightarrow 0^{+}}\left\langle F\left(U_{\varepsilon}\right), \varphi\right\rangle=\langle F(D), \varphi\rangle
$$

for any test function $\varphi$ with support contained in a neighbourhood of $\sigma_{j}$. If $\phi$ is an arbitrary test function, then by using a decomposition as (2.15) we prove (2.18) for $\phi$.

REMARK 2.2. In view of the Dominated Convergence Theorem we can replace the requirement of the uniform convergence of $U_{\varepsilon}$ in Theorems 2.4 and 2.5 by the pointwise convergence $U_{\varepsilon}(\xi) \rightarrow V(\xi)$ a.e. $\xi \in I_{j}$ together with the uniform boundedness of $U_{\varepsilon}$ in $L_{\mathrm{loc}}^{\infty}\left(I_{j}\right)$.

At last, we show that the distribution $D$ obtained as the weak limit of viscosity wavefans in Theorem 2.4 is a weak solution of (1.4)-(1.5) provided that the distribution $F(D)$ is defined by $(2.7)$.

Theorem 2.6. Let $D$ and $F(D)$ be defined by (2.6) and (2.7) respectively. Then

$$
\langle\dot{F}(D)-\xi \dot{D}, \phi\rangle=0 ; \quad \phi \in C_{0}^{\infty}(\mathbf{R}) .
$$

Proof. Let $\sigma_{j} \in\left\{\sigma_{1}, \sigma_{2}, \ldots, \sigma_{k}\right\}$ be fixed and let $\varphi \in C_{0}^{\infty}(\mathbf{R})$ be such that $\operatorname{supp} \varphi \subset$ $I=[a, b]$, with $\sigma_{j} \in I$ and $\sigma_{i} \notin[a, b]$ if $i \neq j$.

Since $\langle\dot{F}(D), \varphi\rangle=-\langle F(D), \dot{\varphi}\rangle$ and $\dot{F}(V)=\xi \dot{V}$ on $\left[a, \sigma_{j}\right) \cup\left(\sigma_{j}, b\right]$, we obtain

$$
\langle\dot{F}(D), \varphi\rangle=\varphi\left(\sigma_{j}\right)\left[F\left(V\left(\sigma_{j}^{+}\right)\right)-F\left(V\left(\sigma_{j}^{-}\right)\right)\right]+\int_{a}^{b} \xi \varphi \dot{V} d \xi-C_{j} \sigma_{j} \dot{\varphi}\left(\sigma_{j}\right)
$$


On the other hand, since $\langle\xi \dot{D}, \varphi\rangle=\langle\dot{D}, \xi \varphi\rangle=-\left\langle D, \frac{d}{d \xi}[\xi \varphi]\right|$, we have

$$
\begin{aligned}
-\langle\xi \dot{D}, \varphi\rangle & =[\xi V \varphi]_{a}^{\sigma_{j}}+[\xi V \varphi]_{\sigma_{j}}^{b}-\int_{a}^{b} \xi \varphi \dot{V} d \xi+\left.C_{j} \frac{d}{d \xi}[\xi \varphi]\right|_{\xi=\sigma_{j}} \\
& =-\varphi\left(\sigma_{j}\right)\left[F\left(V\left(\sigma_{j}^{+}\right)\right)-F\left(V\left(\sigma_{j}^{-}\right)\right)\right]-\int_{a}^{b} \xi \varphi \dot{V} d \xi+C_{j} \sigma_{j} \dot{\varphi}\left(\sigma_{j}\right) \\
& =-\langle\dot{F}(D), \varphi\rangle .
\end{aligned}
$$

We have proved (2.19) for $\varphi$. However, if $\phi$ is an arbitrary function in $C_{0}^{x}(\mathbf{R})$ then, using a decomposition as (2.15) we prove (2.19) for $\phi$.

3. Solution of the Riemann problem. In this section we present the weak solution of (1.6)-(1.7) under the conditions (1.8). This solution is in self-similar form $\left(u\left(\frac{x}{t}\right), v\left(\frac{x}{t}\right)\right)$ where $(u(\xi), v(\xi))$ is a weak solution of the boundary value problem:

$$
\begin{aligned}
& \left\{\begin{array}{l}
\dot{f}(u)=\xi \dot{u}, \\
\frac{d}{d \xi}[v g(u)]=\xi \dot{v}
\end{array} ; \quad \xi \in(-\infty,+\infty),\right. \\
& \left\{\begin{array}{l}
(u(-\infty), v(-\infty))=\left(u_{l}, v_{l}\right), \\
(u(+\infty), v(+\infty))=\left(u_{r}, v_{r}\right) .
\end{array}\right.
\end{aligned}
$$

The eigenvalues and the corresponding right eigenvectors of (1.6) are

$$
\lambda_{1}=f^{\prime}(u), \quad r_{1}=\left(1, \frac{v g^{\prime}(u)}{f^{\prime}(u)-g(u)}\right)^{\mathrm{T}}, \quad \lambda_{2}=g(u), \quad r_{2}=(0,1)^{\mathrm{T}} .
$$

In view of (1.8) the system (1.6) is strictly hyperbolic $\left(\lambda_{1}<\lambda_{2}\right)$, the 1-characteristic family is genuinely nonlinear $\left(\nabla \lambda_{1} \cdot r_{1}>0\right)$, and the 2-characteristic family is linearly degenerate $\left(\nabla \lambda_{2} \cdot r_{2}=0\right)$.

For each left state $\left(u_{l}, v_{l}\right)$ the Hugoniot locus $H\left(u_{l}, v_{l}\right)$ is the curve passing at $\left(u_{l}, v_{l}\right)$ formed by all points $(u, v)$ satisfying

$$
\left\{\begin{array}{l}
s\left(u-u_{l}\right)=f(u)-f\left(u_{l}\right) \\
s\left(v-v_{l}\right)=v g(u)-v_{l} g\left(u_{l}\right)
\end{array}\right.
$$

for some $s \in \mathbf{R}$.

The portion of $H\left(u_{l}, v_{l}\right)$ used to build the classical weak solutions of (1.6)-(1.7) involving discontinuities is the subset $S_{1}\left(u_{l}, v_{l}\right) \cup D_{2}\left(u_{l}, v_{l}\right)$ where

$$
D_{2}\left(u_{l}, v_{l}\right)=\left\{\left(u_{l}, v\right): v \in \mathbf{R}\right\}
$$

is the 2-contact discontinuity curve and

$$
S_{1}\left(u_{l}, v_{l}\right)=\left\{(u, v): v=v_{l} \frac{s(u)-\lambda_{2}\left(u_{l}\right)}{s(u)-\lambda_{2}(u)}, u \leq u_{l}, s(u)<\lambda_{2}(u)\right\}
$$

is the 1-shock curve. Here $s(u)=\frac{f(u)-f\left(u_{l}\right)}{u-u_{l}}$ is the shock velocity. 
REMARK 3.1. Since $s\left(u_{l}\right)<\lambda_{2}\left(u_{l}\right)$, the function $s(u)-\lambda_{2}(u)$ is negative on an interval that contains $u_{l}$. However, if $-\infty<u_{l}^{*}<u_{l}$ is the first zero of this function to the left of $u_{l}$, then $S_{1}\left(u_{l}, v_{l}\right)$ tends to the line $u=u_{l}^{*}$ when $u \rightarrow\left(u_{l}^{*}\right)^{-}$, i.e., its $v$-coordinate becomes unbounded.

The 1-rarefaction curve $R_{1}\left(u_{l}, v_{l}\right)$ is the portion of the integral curve in the direction of $r_{1}$ in which $\lambda_{1}$ increases. Thus,

$$
R_{1}\left(u_{l}, v_{l}\right)=\left\{(u, v): v=v_{l} \exp \left(-\int_{u_{l}}^{u} \frac{g^{\prime}(z)}{\lambda_{2}(z)-\lambda_{1}(z)} d z\right), u \geq u_{l}\right\} .
$$

The rarefaction curve corresponding to the eigenvalue $\lambda_{2}$ does not exist since $\lambda_{2}$ is constant on the integral curve in the direction of $r_{2}$, which coincides with $D_{2}\left(u_{l}, v_{l}\right)$.

The classical solution of (1.6)-(1.7) is obtained by using the curves $S_{1}\left(u_{l}, v_{l}\right), R_{1}\left(u_{l}, v_{l}\right)$, and $D_{2}\left(u_{r}, v_{r}\right)$.

If $u_{r}>u_{l}$ the solution of (1.6)-(1.7) is the composition $R_{1}+D_{2}$ that consists of the rarefaction wave $R_{1}$ connecting $\left(u_{l}, v_{l}\right)$ to $\left(u_{r}, v_{m}\right)$ followed by the contact discontinuity $D_{2}$ connecting $\left(u_{r}, v_{m}\right)$ to $\left(u_{r}, v_{r}\right)$. The intermediate state $\left(u_{r}, v_{m}\right)$ is the unique point in $R_{1}\left(u_{l}, v_{l}\right) \cap D_{2}\left(u_{r}, v_{r}\right)$. This solution can be written in the following self-similar form:

$$
\begin{gathered}
u(\xi)= \begin{cases}u_{l} ; & \xi \leq \lambda_{1}\left(u_{l}\right), \\
\lambda_{1}^{-1}(\xi) ; & \lambda_{1}\left(u_{l}\right) \leq \xi \leq \lambda_{1}\left(u_{r}\right), \\
u_{r} ; & \xi \geq \lambda_{1}\left(u_{r}\right),\end{cases} \\
v(\xi)= \begin{cases}v_{l} \exp \left(-\int_{u_{l}}^{u(\xi)} \frac{g^{\prime}(z)}{\lambda_{2}(z)-\lambda_{1}(z)} d z\right) ; & \xi<\lambda_{2}\left(u_{r}\right), \\
v_{r} ; & \xi>\lambda_{2}\left(u_{r}\right) .\end{cases}
\end{gathered}
$$

If $u_{r}<u_{l}$ and $s\left(u_{r}\right)<\lambda_{2}\left(u_{r}\right)$ the solution of (1.6)-(1.7) is the composition $S_{1}+D_{2}$ that consists of the shock wave $S_{1}$ connecting $\left(u_{l}, v_{l}\right)$ to $\left(u_{r}, v_{m}\right)$ followed by the contact discontinuity $D_{2}$ connecting $\left(u_{r}, v_{m}\right)$ to $\left(u_{r}, v_{r}\right)$, where the intermediate state $\left(u_{r}, v_{m}\right)$ is the unique point in $S_{1}\left(u_{l}, v_{l}\right) \cap D_{2}\left(u_{r}, v_{r}\right)$. In the self-similar form this solution becomes:

$$
\begin{gathered}
u_{s}(\xi)= \begin{cases}u_{l} ; & \xi<s, \\
u_{r} ; & \xi>s,\end{cases} \\
v(\xi)= \begin{cases}v_{l} ; & \xi<s, \\
v_{m} ; & s<\xi<\lambda_{2}\left(u_{r}\right), \\
u_{r} ; & \xi>\lambda_{2}\left(u_{r}\right),\end{cases}
\end{gathered}
$$

where $s=s\left(u_{r}\right)$ and $v_{m}=v_{l}\left(\frac{s\left(u_{r}\right)-\lambda_{2}\left(u_{l}\right)}{s\left(u_{r}\right)-\lambda_{2}\left(u_{r}\right)}\right)$.

If $u_{r}<u_{l}$ and $s\left(u_{r}\right) \geq \lambda_{2}\left(u_{r}\right)$ then, according to Remark 3.1 above, the right state $\left(u_{r}, v_{r}\right)$ cannot belong to $S_{1}\left(u_{l}, v_{l}\right)$ or $R_{1}\left(u_{l}, v_{l}\right)$. Henceforth, in this case there exists no standard weak solution for (1.6)-(1.7). So, we solve the problem as in [13] using the delta-shock wave $S_{\delta}$ which we present below as being a distribution of the self-similar form (2.6) satisfying a condition of overcompressivity. With this condition we obtain uniqueness of solutions in the class of the simple waves and delta-shock waves. 
Definition 3.1. Let $S_{\delta}$ be a distribution of the form:

$$
S_{\delta}=\left(u_{s}(\xi), v_{s}(\xi)+c \delta_{s}\right)
$$

where $s$ is a real number, $u_{s}$ is a function as in (3.6),

$$
v_{s}(\xi)= \begin{cases}v_{l} ; & \xi<s \\ v_{r} ; & \xi>s\end{cases}
$$

and

$$
c=s\left[v_{r}-v_{l}\right]-\left[v_{r} g\left(u_{r}\right)-v_{l} g\left(u_{l}\right)\right] .
$$

Then $S_{\delta}$ is called a delta-shock wave for (3.1)-(3.2) if the following two conditions are satisfied:

(i) the $S_{\delta}$-Rankine-Hugoniot condition

$$
s\left[u_{r}-u_{l}\right]=\left[f\left(u_{r}\right)-f\left(u_{l}\right)\right]
$$

(ii) the $S_{\delta}$-entropy condition

$$
\lambda_{2}\left(u_{r}\right) \leq s \leq \lambda_{1}\left(u_{l}\right)
$$

Some remarks are in order.

1. According to [13] we define $u_{s}$ at $s$ such that $\lambda_{2}\left(u_{s}(s)\right)=s$. So, from Theorem 2.6, a delta-shock $S_{\delta}$ is a solution of (3.1)-(3.2) in the sense of distributions if the product $c \delta_{s} \cdot g\left(u_{s}\right)$ is defined by

$$
\left\langle c \delta_{s} \cdot g\left(u_{s}\right), \phi\right\rangle:=c g\left(u_{s}(s)\right) \phi(s)=c s \phi(s)=\left\langle s c \delta_{s}, \phi\right\rangle ; \quad \phi \in C_{0}^{\infty}(\mathbf{R}),
$$

which is a Radon measure.

2. In view of (1.8) and of (3.3) a delta-shock $S_{\delta}$ is an overcompressive wave, i.e., all characteristics enter on the discontinuity.

3. The inequality $s \leq \lambda_{l}\left(u_{l}\right)$ in (3.11) is equivalent to $u_{l}>u_{r}$ because of the convexity condition on $f$ in (1.8). So, the following two conditions characterize a delta-shock $S_{\delta}$ :

$$
u_{l}>u_{r} \quad \text { and } \quad \lambda_{2}\left(u_{r}\right) \leq s .
$$

4. Without (3.11), the distribution $S_{\delta}$ could be another weak solution than $S_{1}+R_{1}$ when $s\left(u_{r}\right)<\lambda_{2}\left(u_{r}\right)$.

5. Taking $\xi=x / t$ and denoting $S_{\delta}$ by $S_{\delta . x . t}$ we can write

$$
S_{\delta . x . t}=\left(u_{s}\left(\frac{x}{t}\right), v_{s}\left(\frac{x}{t}\right)+v_{\delta}\right)
$$

where the distribution $v_{\delta}$ is defined by

$$
\left\langle v_{\delta}, \phi\right\rangle=c \int_{-\infty}^{+\infty} t \phi(s t, t) d t ; \quad \phi \in C_{0}^{\infty}\left(\mathbf{R}_{+}^{2}\right) .
$$

So, $v_{\delta}$ is the Dirac-delta with weight $c$ supported on the discontinuity $x=s t$. 
4. Existence, uniqueness and estimates. In this section we show existence, uniqueness and estimates in $L^{\infty}$ and $L_{\text {loc }}^{1}$ for the smooth solutions of the boundary value problem:

$$
\begin{aligned}
\left\{\begin{array}{l}
e \ddot{u}=\dot{f}(u)-\xi \dot{u} ; \\
e \ddot{v}=\frac{d}{d \xi}[v g(u)]-\xi \dot{v},
\end{array} \quad \xi \in(-\infty,+\infty),\right. \\
\left\{\begin{array}{l}
(u(-\infty), v(-\infty))=\left(u_{l}, v_{l}\right), \\
(u(+\infty), v(+\infty))=\left(u_{r}, v_{r}\right) .
\end{array}\right.
\end{aligned}
$$

We assume that $f(u)$ and $g(u)$ are smooth functions with $g^{\prime}(u)>0$ for all $u$ on the closed interval with extremes $u_{l}$ and $u_{r}$, which we denote by $I\left[u_{l}, u_{r}\right]$.

REMARK 4.1. All results of this section remain true, with small changes in the proofs, if we replace the second equation in (4.1) by the following nonlinear equation:

$$
e \ddot{v}=\frac{d}{d \xi}[v g(u, v)+h(v)]-\xi \dot{v},
$$

where $h(v)$ and $g(u, v)$ are smooth functions with $g_{u}>0$ on $I\left[u_{l}, v_{l}\right]$, and $g(J, \mathbf{R})$ is a bounded set for each closed interval $J$.

THEOREM 4.1. Let $\left(u_{1}(\xi), v_{1}(\xi)\right)$ and $\left(u_{2}(\xi), v_{2}(\xi)\right)$ be two smooth solutions of $(4.1)-$ (4.2). Then $u_{1}=u_{2}$ and $v_{1}=v_{2}$.

Proof. First we prove that $u_{1}=u_{2}$ by showing that $w:=u_{2}-u_{1}$ is null everywhere. We can verify that $w$ is a smooth solution of the boundary value problem

$$
\left\{\begin{array}{l}
\varepsilon \ddot{w}=\frac{d}{d \xi}[w h]-\xi \dot{w} ; \quad \xi \in(-\infty,+\infty), \\
w(-\infty)=0, \quad w(+\infty)=0
\end{array}\right.
$$

where $h(\xi)=\int_{0}^{1} f^{\prime}\left(u_{2}(\xi) \theta+(1-\theta) u_{1}(\xi)\right) d \theta$. (We observe that $h$ is a bounded function.)

Let us suppose that $w$ is not the null function. Let $\alpha$ and $\beta$ be consecutive zeros of $w$ with $-\infty \leq \alpha<\beta \leq+\infty$. So, integrating (4.3) by parts on $(\alpha, \beta)$ we find

$$
\varepsilon(\dot{w}(\beta)-\dot{w}(\alpha))=\int_{\alpha}^{\beta} w d \xi
$$

(If $\alpha=-\infty$ and/or $\beta=+\infty$ then we turn to Remark 2.1 to verify that $\lim _{\xi \rightarrow \alpha^{-}} \xi w(\xi)=$ $0=\lim _{\xi \rightarrow \beta^{+}} \xi w(\xi)$.)

Now, if $w>0$ on $(\alpha, \beta)$, then $\dot{w}(\beta) \leq 0 \leq \dot{w}(\alpha)$ and $\int_{\alpha}^{\beta} w d \xi>0$; and if $w<0$ on $(\alpha, \beta)$, then $\dot{w}(\beta) \geq 0 \geq \dot{u}(\alpha)$ and $\int_{\alpha}^{\beta} w d \xi<0$. In both cases we have a contradiction with (4.4). So, we must have $w \equiv 0$.

Similarly we prove that $v:=v_{2}-v_{1}$ is null everywhere since $v$ is a smooth solution of the boundary value problem (4.3) with $h=g\left(u_{1}\right)=g\left(u_{2}\right)$.

To prove existence of a solution to (4.1)-(4.2) using Dafermos' Theorem stated in Sec. 2 (Theorem 2.2) we need to show that all smooth solutions $(u(\xi), v(\xi))$ of the following two-parameter boundary value problem

$$
\left\{\begin{array}{l}
\varepsilon \ddot{u}=\mu \dot{f}(u)-\xi \dot{u}, \\
\varepsilon \ddot{v}=\mu \frac{d}{d \xi}[v g(u)]-\xi \dot{v}, \quad \xi \in(-L, L),
\end{array}\right.
$$




$$
\left\{\begin{array}{l}
(u(-L), v(-L))=\mu\left(u_{l}, v_{l}\right), \\
(u(L), v(L))=\mu\left(u_{r}, v_{r}\right) .
\end{array}\right.
$$

satisfy the a priori $L^{\infty}$-estimate:

$$
\sup _{\xi \in(-L . L)}\|(u(\xi), v(\xi))\| \leq M
$$

where the positive constant $M$ must be independent of the parameters $L \geq 1$ and $\mu \in$ $[0,1]$.

REMARK 4.2. The solutions of the first equation in (4.5) are monotonic. In fact, if $u$ is a smooth solution of $\varepsilon \ddot{u}=\mu \dot{f}(u)-\xi \dot{u}$ on an interval $I$ then, by integration, we can verify that

$$
\dot{u}(\xi)=\dot{u}\left(\xi_{0}\right) \exp \left(-\frac{1}{\varepsilon} \int_{\xi_{0}}^{\xi}\left[\mu f^{\prime}(u(\eta))-\eta\right] d \eta\right)
$$

for any $\xi_{0} \in I$.

As a consequence of Remark 4.2, the $u(\xi)$-component of any smooth solution of (4.5)(4.6) satisfies

$$
\sup _{\xi \in(-L . L)}|u(\xi)| \leq \max \left\{\left|\mu u_{l}\right|,\left|\mu u_{r}\right|\right\} \leq \bar{u},
$$

where $\bar{u}=\max \left\{\left|u_{l}\right|,\left|u_{r}\right|\right\}$, which is a constant that does not depend on $L \geq 1$ and $\mu \in[0,1]$.

Therefore, we need only prove that there exists a constant $C$ that is independent of $L$ and $\mu$ such that

$$
\sup _{\xi \in(-L . L)}|v(\xi)| \leq C,
$$

for all solutions $v(\xi)$ of the following linear (considering $g(u(\xi))$ as a coefficient) boundary value problem:

$$
\begin{gathered}
e \ddot{v}=\mu \frac{d}{d \xi}[v g(u)]-\xi \dot{v} ; \quad \xi \in(-L, L), \\
v(-L)=\mu v_{l}, \quad v(L)=\mu v_{r} .
\end{gathered}
$$

LEMma 4.2. Let $v(\xi)$ be a smooth solution of $(4.10)$ on $(-L, L)$. We claim that

(i) if $v(\alpha)=v(\beta)=0$ for any $\alpha, \beta \in(-L, L)$, then $v(\xi)=0$ for all $\xi \in(-L, L)$;

(ii) if $u(-L)<u(L)$, then a critical point $\xi_{0} \in(-L, L)$ of $v$ is a maximum (or minimum) point if and only if $v\left(\xi_{0}\right)<0\left(\right.$ or $\left.v\left(\xi_{0}\right)>0\right)$, and furthermore.

$$
|v(\xi)| \leq \max \{|v(-L)|,|v(L)|\}
$$

(iii) if $u(-L)>u(L)$ then a critical point $\xi_{0} \in(-L, L)$ of $v$ is a maximum (or minimum) point if and only if $v\left(\xi_{0}\right)>0\left(\right.$ or $\left.v\left(\xi_{0}\right)<0\right)$.

Proof. The proof of (i) is based on the same arguments that we have used to prove the uniqueness above. We only observe that if $v(\xi)=0$ for all $\xi \in(\alpha, \beta)$, then $v \equiv 0$ on $(-L, L)$ in view of the classical uniqueness theorems about initial-value problems for 
linear ordinary differential equations. In order to verify (ii) and (iii) we evaluate (4.10) at $\xi=\xi_{0}$ to get

$$
e \ddot{v}\left(\xi_{0}\right)=\mu v\left(\xi_{0}\right) g^{\prime}\left(u\left(\xi_{0}\right)\right) \dot{u}\left(\xi_{0}\right) .
$$

Since $g^{\prime}>0$ and $u$ is a strictly monotonic function, we conclude the proof of statements (ii) and (iii) directly from (4.13).

We observe from Lemma 4.2 that if $u(-L) \neq u(L)$ and $v$ assumes three local extremes, then $v$ vanishes twice, which implies from (i) that $v \equiv 0$. Furthermore, if $u(-L)<u(L)$ and $v(-L), v(L) \leq 0$ (or $v(-L), v(L) \geq 0$ ) then in view of (i), $v$ cannot assume a local minimum (or maximum). Also, if $v(-L) v(L)<0$ then $v$ is strictly monotonic. At last, if $u(-L)>u(L)$ and $v$ assumes two local extremes $\xi_{1}$ and $\xi_{2}$, then $v\left(\xi_{1}\right) v\left(\xi_{2}\right)<0$ and $v$ vanishes only at one $\bar{\xi} \in\left(\xi_{1}, \xi_{2}\right)$.

To prove estimates in $L^{\infty}(\mathbf{R})$ and $L_{\text {loc }}^{1}(\mathbf{R})$ on $v$ we need another lemma.

LEMMA 4.3. Let $v(\xi)$ be a smooth solution of $(4.10)$ on $[a, b] \subset(-L, L)$ with $u(b)>u(a)$. Then

$$
\frac{1}{\beta-\alpha} \int_{\alpha}^{\beta}|v(\eta)| d \eta \leq c\left(1+\frac{k}{\beta-\alpha}\right)
$$

and

$$
\max _{\xi \in[a, b]}|v(\xi)| \leq c e^{k}\left(1+\frac{k}{\varepsilon}\right),
$$

where $c=\max \{|v(a)|,|v(b)|\}$ and $k=(u(a)-u(b)) \max \left\{g^{\prime}(u): u \in[u(b), u(a)]\right\}$.

Proof. We can assume that $v$ is not monotonic. Otherwise, the lemma is trivial. First we prove (4.14). The proof is based on arguments encountered in [2] or in [15] in a more general situation. Initially we suppose that $0 \leq v(a)<v(b)$ or $0 \leq v(b)<v(a)$. So it follows from Lemma 4.2 that $v>0$ on $(a, b)$ and that there exists $\xi_{0} \in(a, b)$ such that $v$ is strictly increasing on $\left(a, \xi_{0}\right)$, it assumes the maximum value in $\xi=\xi_{0}$ and it is strictly decreasing on $\left(\xi_{0}, b\right)$.

Let $a \leq \bar{a}<\xi_{0}<\bar{b} \leq b$ be such that

$$
0<v(\bar{a})=v(\bar{b})=c<v(\eta) ; \quad \eta \in(\bar{a}, \bar{b}) .
$$

We observe that if $0 \leq v(a)<v(b)$, then $a<\bar{a}$ and $\bar{b}=b$, and if $0 \leq v(b)<v(a)$, then $a=\bar{a}$ and $\bar{b}<b$. By considering these two possibilities we obtain

$$
\int_{\alpha}^{\beta}(v(\eta)-c) d \eta \leq \int_{\bar{a}}^{\bar{b}}(v(\eta)-c) d \eta ; \quad a \leq \alpha<\beta \leq b .
$$

Now, for each $\xi \in\left[\bar{a}, \xi_{0}\right)$ let $\xi^{\prime} \in\left(\xi_{0}, \bar{b}\right]$ be the unique number satisfying

$$
0<v(\xi)=v\left(\xi^{\prime}\right)<v(\eta) ; \quad \eta \in\left(\xi, \xi^{\prime}\right) .
$$

Thus, integrating $(4.10)$ by parts on $\left[\xi, \xi^{\prime}\right]$ we get

$$
\varepsilon\left(\dot{v}\left(\xi^{\prime}\right)-\dot{v}(\xi)\right)=\mu v(\xi)|g(u)|_{\xi}^{\xi^{\prime}}+\int_{\xi}^{\xi^{\prime}}[v(\eta)-v(\xi)] d \eta
$$


Since $\dot{v}\left(\xi^{\prime}\right) \leq 0 \leq \dot{v}(\xi)$ we have

$$
\int_{\xi}^{\xi^{\prime}}[v(\eta)-v(\xi)] d \eta \leq \mu v(\xi)\left(g(u(\xi))-g\left(u\left(\xi^{\prime}\right)\right) \leq k v(\xi) .\right.
$$

So, if $\xi=\bar{a}$ then $\xi^{\prime}=\bar{b}$ and $\int_{\bar{a}}^{\bar{b}}[v(\eta)-c] d \eta \leq k c$ (because $v(\bar{a})=v(\bar{b})=c$ ). Therefore, it follows from (4.17) that

$$
\int_{\alpha}^{\beta}(v(\eta)-c) d \eta \leq k c ; \quad a \leq \alpha<\beta \leq b .
$$

Dividing this last inequality by $\beta-\alpha$ we obtain immediately (4.14) since $v \geq 0$.

On the other hand, if $\xi \in\left(\bar{a}, \xi_{0}\right)$ we have

$$
\varepsilon \dot{v}(\xi) \leq \varepsilon \dot{v}\left(\xi^{\prime}\right)-\mu v(\xi)[g(u)]_{\xi}^{\xi^{\prime}} \leq \mu v(\xi)[g(u)]_{\xi^{\prime}}^{\xi} \leq k v(\xi)
$$

since $\int_{\xi}^{\xi^{\prime}}[v(\eta)-v(\xi)] \geq 0$.

We have concluded that $v$ satisfies the differential inequality $\dot{v} \leq \frac{k v}{\varepsilon}$ on $\left(\bar{a}, \xi_{0}\right)$, which after an integration gives us

$$
v\left(\xi_{0}\right)<v(\xi) \exp \left(\frac{k}{\varepsilon}\left(\xi_{0}-\xi\right)\right) ; \quad \xi \in\left(\bar{a}, \xi_{0}\right) .
$$

If $\xi_{0}-\bar{a} \leq \varepsilon$ we evaluate (4.19) at $\xi=\bar{a}$ to find

$$
\max _{\xi \in[a, b]}|v(\xi)|=v\left(\xi_{0}\right) \leq c \mathrm{e}^{k} \leq c \mathrm{e}^{k}\left(1+\frac{k}{\varepsilon}\right) .
$$

Otherwise, if $\xi_{0}-\bar{a}>\varepsilon$ then we take $\tilde{\xi} \in\left(\bar{a}, \xi_{0}\right)$ such that $\xi_{0}-\tilde{\xi}=\varepsilon$. Thus, evaluating (4.19) at $\xi=\tilde{\xi}$ we have

$$
\max _{\xi \in[a, b]}|v(\xi)|=v\left(\xi_{0}\right)<v(\tilde{\xi}) \exp \left(\frac{k}{\varepsilon}\left(\xi_{0}-\tilde{\xi}\right)\right) \leq v(\tilde{\xi}) \mathrm{e}^{k} .
$$

Since $v$ is strictly increasing on $\left[\tilde{\xi}, \xi_{0}\right)$ we get

$$
\max _{\xi \in[a, b]}|v(\xi)|<\frac{\mathrm{e}^{k}}{\xi_{0}-\tilde{\xi}} \int_{\tilde{\xi}}^{\xi_{0}} v(\eta) d \eta \leq c \mathrm{e}^{k}\left(1+\frac{k}{\varepsilon}\right) .
$$

We have finished the proof when $v(a)$ and $v(b)$ are both nonnegative and consequently, in view of the linearity of (4.10), also when $v(a)$ and $v(b)$ are both nonpositive. Now, if $v(a) v(b)<0$ then there exists $\bar{\xi} \in(a, b)$ such that $v(\bar{\xi})=0$. By using (4.14) and (4.15) on intervals $[a, \bar{\xi}]$ and $[\bar{\xi}, b]$ we complete this proof.

Next, assuming that $u_{l}>u_{r}$ we prove the existence of viscosity wavefans and give two estimates for these functions. For the case $u_{l} \leq u_{r}$ the existence of smooth solutions of (4.1)-(4.2) follows directly from Theorem 2.2 (Sec. 2) in view of (4.9) and (ii) from Lemma 4.2. Furthermore, these solutions are uniformly bounded in the sup norm and in total variation.

Theorem 4.4. For each $\varepsilon>0$ there exists a unique smooth solution $\left(u_{\varepsilon}, v_{\varepsilon}\right)$ of the boundary value problem (4.1)-(4.2) with $u_{l}>u_{r}$. Furthermore, 
(i) there exists a positive constant $M$, independent of $\varepsilon$, such that

$$
\left\|u_{\varepsilon}\right\|_{\infty} \leq M, \quad\left\|v_{\varepsilon}\right\|_{\infty} \leq \frac{M}{\varepsilon} ;
$$

(ii) for each closed interval $I$, there exists a positive constant $K_{I}$, independent of $\varepsilon$, such that

$$
\int_{I}\left|v_{\varepsilon}(\xi)\right| d \xi \leq K_{I}
$$

Proof. The uniqueness was proved in Theorem 4.1. In order to prove the existence through Theorem 2.2 we need to verify (4.7) for any solution $(u(\xi), v(\xi))$ of $(4.5)-(4.6)$. In view of (4.8) we need only to prove (4.9) for a constant $C$ that must be independent of $L$ and $\mu$. But, taking $a \rightarrow-L$ and $b \rightarrow L$ in Lemma 4.3 we have

$$
\sup _{\xi \in(-L, L)}|v(\xi)| \leq c \mathrm{e}^{k}\left(1+\frac{k}{\varepsilon}\right)
$$

where $c=\mu \max \left\{\left|v_{l}\right|,\left|v_{r}\right|\right\}$ and $k=\mu\left(u_{l}-u_{r}\right) \max \left\{g^{\prime}(u): u \in\left[\mu u_{r}, \mu u_{l}\right]\right\}$.

By taking $\bar{v}=\max \left\{\left|v_{l}\right|,\left|v_{r}\right|\right\}$ and $\bar{u}=\max \left\{\left|u_{l}\right|,\left|u_{r}\right|\right\}$ we have $c \leq \bar{v}$ and $k \leq \bar{k}:=$ $\left(u_{l}-u_{r}\right) \max \left\{g^{\prime}(u):|u| \leq \bar{u}\right\}$. Therefore, we obtain (4.9) with $C=\varepsilon^{-1} \mathrm{e}^{\bar{k}} \bar{v}(1+\bar{k})$ and conclude, via Theorem 2.2, that there exists a smooth solution $\left(u_{\varepsilon}, v_{\varepsilon}\right)$ of $(4.1)-(4.2)$ satisfying the same bounds (4.8) and (4.9) with $L=\infty$. We also obtain (4.20) by taking $M=\max \left\{\bar{u}, \bar{v} \mathrm{e}^{\bar{k}}(1+\bar{k})\right\}$.

To prove (4.21) we fix a closed interval $I=[\alpha, \beta]$ and take $L>0$ such that $I \subset(-L, L)$. Taking $\mu=1$ in Lemma 4.3 and noting that $u_{\varepsilon}(-L)>u_{\varepsilon}(L)$ we have

$$
\int_{I}\left|v_{\varepsilon}(\eta)\right| d \eta \leq c_{\varepsilon}(L)\left(\beta-\alpha+k_{\varepsilon}(L)\right)
$$

where $k_{\varepsilon}(L)=\left(u_{\varepsilon}(-L), u_{\varepsilon}(L)\right) \max \left\{g^{\prime}(u): u \in\left[u_{\varepsilon}(L), u_{\varepsilon}(-L)\right]\right\}$ and $c_{\varepsilon}(L)=$ $\max \left\{\left|v_{\varepsilon}(-L)\right|,\left|v_{\varepsilon}(L)\right|\right\}$.

Now, when $L \rightarrow \infty$ we have that $c_{\varepsilon}(L) \rightarrow \bar{v}$ and

$$
k_{\varepsilon}(L) \rightarrow\left(u_{l}-u_{r}\right) \max \left\{g^{\prime}(u): u \in\left[u_{r}, u_{l}\right]\right\} \leq \bar{k} .
$$

Therefore, we have proved (4.21) with $K_{I}=\bar{v}(\beta-\alpha+\bar{k})$.

5. Convergence. In this section we prove the weak convergence of the viscosity wavefans associated with the Riemann problem (1.6)-(1.7) for $u_{l}>u_{r}$. We assume the conditions in (1.8) on $f$ and $g$.

In the following $\left(u_{\varepsilon}, v_{\varepsilon}\right)$ denotes the viscosity wavefans in the self-similar form, i.e., the solutions of (4.1)-(4.2) that, in conforming with Sec. 4, are unique for each $\varepsilon>0$. Also, $u_{s}$ and $v_{s}$ denote respectively the discontinuities (3.6) and (3.8) at $\xi=s$ where

$$
s=\frac{f\left(u_{r}\right)-f\left(u_{l}\right)}{u_{r}-u_{l}} .
$$

We begin by stating a result that can be found in [13]. 
LEmma 5.1. For each $\varepsilon>0$ there exists a unique $\xi_{\varepsilon}$ such that $\xi_{\varepsilon}=\lambda_{1}\left(u_{\varepsilon}\left(\xi_{\varepsilon}\right)\right)$ and $\lim _{\varepsilon \rightarrow 0} \xi_{\varepsilon}=s$. Furthermore

(a) there exists a constant $M_{1}$, independent of $\varepsilon$, such that

$$
\left|\dot{u}_{\varepsilon}(\xi)\right| \leq \frac{M_{1}}{\varepsilon} \exp \left(-\frac{1}{2 \varepsilon}\left(\xi-\xi_{\varepsilon}\right)^{2}\right) ; \quad \xi \in(-\infty,+\infty) ;
$$

(b) $u_{\varepsilon}$ converges to $u_{s}$ uniformly on $(-\infty, s-\alpha] \cup[s+\alpha, \infty)$ for any $\alpha>0$.

In view of Lemma 5.1 we can apply Theorem 2.4 (Sec. 2) to verify the weak convergence $u_{\varepsilon} \rightarrow u_{s}$.

Next we pursue the weak convergence of $v_{\varepsilon}$. For this we will return to Lemma 5.1 later.

Proposition 5.2. For each $\varepsilon>0$ there exists a unique $\eta_{\varepsilon}$ such that

$$
\eta_{\varepsilon}=\lambda_{2}\left(u_{\varepsilon}\left(\eta_{\varepsilon}\right)\right)
$$

Furthermore, $\xi_{\varepsilon}<\eta_{\varepsilon}$ and

$$
\lim _{\varepsilon \rightarrow 0} \eta_{\varepsilon}=\max \left\{s, \lambda_{2}\left(u_{r}\right)\right\}
$$

Proof. Since $\lambda_{2}\left(u_{\varepsilon}\right)$ is a decreasing smooth function in $(-\infty,+\infty)$ (because $g^{\prime}>0$ and $\dot{u}_{\varepsilon}<0$ ) it has a unique fixed point $\eta_{\varepsilon}$. From (1.8) it follows that $\xi_{\varepsilon}<\eta_{\varepsilon}$.

Since $\eta_{\varepsilon}$ is a bounded sequence, it suffices to prove that all convergent subsequences of $\eta_{\varepsilon}$ converge to $\max \left\{s, \lambda_{2}\left(u_{r}\right)\right\}$. So, let $\eta_{\varepsilon_{n}}$ be a subsequence converging to some $\eta_{0}$. Taking $n \rightarrow \infty$ on inequality $\xi_{\varepsilon_{n}}<\eta_{\varepsilon_{n}}$ we have from Lemma 5.1 that $s \leq \eta_{0}$.

If $s<\eta_{0}$ then from Lemma 5.1 (item (b)) it follows that $u_{\varepsilon_{n}}\left(\eta_{\varepsilon_{n}}\right) \rightarrow u_{r}$ and so from (5.3) we have $s<\eta_{0}=\lambda_{2}\left(u_{r}\right)$. Therefore, in this case $\eta_{0}=\max \left\{s, \lambda_{2}\left(u_{r}\right)\right\}$.

If $s=\eta_{0}$ we must have again $\eta_{0}=\max \left\{s, \lambda_{2}\left(u_{r}\right)\right\}$ because, otherwise, if $s<\lambda_{2}\left(u_{r}\right)$ then $s<\lambda_{2}\left(u_{r}\right)<\lambda_{2}\left(u_{\varepsilon_{n}}\left(\eta_{\varepsilon_{n}}\right)\right)=\eta_{\varepsilon_{n}}$ and taking $n \rightarrow \infty$ we could arrive at the contradiction $s<\lambda_{2}\left(u_{r}\right) \leq \eta_{0}$.

Now we prove an estimate for $\dot{v}_{\varepsilon}$ in terms of the auxiliary function

$$
E_{\varepsilon}(\xi):=\max \left\{\exp \left(-\frac{1}{2 \varepsilon}\left(\xi-\xi_{\varepsilon}\right)^{2}\right), \exp \left(-\frac{1}{2 \varepsilon}\left(\xi-\eta_{\varepsilon}\right)^{2}\right)\right\} ; \quad \xi \in(-\infty,+\infty) .
$$

Proposition 5.3. There exists a constant $C$, independent of $\varepsilon$, such that

$$
\left|\dot{v}_{\varepsilon}(\xi)\right| \leq \frac{C}{\varepsilon^{3}} E_{\varepsilon}(\xi)\left(1+\left|\xi-\eta_{\varepsilon}\right|\right), \quad \xi \in \mathbf{R} .
$$

Proof. Integrating the second equation in (4.1) we obtain

$$
\dot{v}_{\varepsilon}(\xi)=a_{\varepsilon}(\xi)+b_{\varepsilon}(\xi) ; \quad \xi \in(-\infty,+\infty),
$$

where $a_{\varepsilon}(\xi)=\dot{v}_{\varepsilon}\left(\eta_{\varepsilon}\right) \exp \left(\frac{1}{\varepsilon} \int_{\eta_{\varepsilon}}^{\xi}\left[\lambda_{2}\left(u_{\varepsilon}(\eta)\right)-\eta\right] d \eta\right)$ and

$$
b_{\varepsilon}(\xi)=\frac{1}{\varepsilon} \int_{\eta=}^{\xi} v_{\varepsilon}(\tau) \dot{\lambda}_{2}\left(u_{\varepsilon}(\tau)\right) \exp \left(\frac{1}{\varepsilon} \int_{\tau}^{\xi}\left[\lambda_{2}\left(u_{\varepsilon}(\eta)\right)-\eta\right] d \eta\right) d \tau
$$


From the monotonicity of $\lambda_{2}\left(u_{\varepsilon}\right)$ and from the definition of $\eta_{\varepsilon}$ it follows that

$$
\exp \left(\frac{1}{\varepsilon} \int_{\eta_{\varepsilon}}^{\xi}\left[\lambda_{2}\left(u_{\xi}(\eta)\right)-\eta\right] d \eta\right) \leq \exp \left(-\frac{1}{2 \varepsilon}\left(\xi-\xi_{\varepsilon}\right)^{2}\right) \leq E_{\varepsilon}(\xi)
$$

for all $\xi \in(-\infty,+\infty)$.

From (4.20) we have $\left\|\left(u_{\varepsilon}, v_{\varepsilon}\right)\right\|_{\infty}=O(1 / \varepsilon)$ and $\left\|\left(f\left(u_{\varepsilon}\right), v_{\varepsilon} \lambda_{2}\left(u_{\varepsilon}\right)\right)\right\|_{\infty}=O(1 / \varepsilon)$. Hence, taking $I=\left[\lambda_{2}\left(u_{r}\right), \lambda_{2}\left(u_{l}\right)\right]$ and $p=1$ in Remark 2.1 it follows that $\left|\dot{v}_{\varepsilon}(\xi)\right| \leq C_{1} / \varepsilon^{2}$ for all $\xi \in I$, where $C_{1}$ depends only on $\lambda_{2}\left(u_{l}\right)$ and $\lambda_{2}\left(u_{r}\right)$. Since $\eta_{\varepsilon} \in I$, we have $\left|\dot{v}_{\varepsilon}\left(\eta_{\varepsilon}\right)\right| \leq C_{1} / \varepsilon^{2}$ and so

$$
\left|a_{\varepsilon}(\xi)\right| \leq \frac{C_{1}}{\varepsilon^{2}} E_{\varepsilon}(\xi) ; \quad \xi \in(-\infty,+\infty) .
$$

On the other hand, from Lemma 5.1 (item (a)) and (4.20) we get

$$
\begin{aligned}
\left|b_{\varepsilon}(\xi)\right| & \leq \frac{K M M_{1}}{\varepsilon^{3}}\left|\int_{\eta_{\varepsilon}}^{\xi} \exp \left(\frac{1}{\varepsilon} \int_{\tau}^{\xi}\left[\lambda_{2}\left(u_{\varepsilon}(\eta)\right)-\eta\right] d \eta-\frac{1}{2 \varepsilon}\left(\tau-\xi_{\varepsilon}\right)^{2}\right) d \tau\right| \\
& =\frac{C_{2}}{\varepsilon^{3}}\left|\int_{\eta_{\varepsilon}}^{\xi} F_{\varepsilon}(\xi, \tau) d \tau\right|,
\end{aligned}
$$

where $K=\max \left\{g^{\prime}(u): u \in\left[u_{r}, u_{l}\right]\right\}, C_{2}=K M M_{1}$, and

$$
0<F_{\varepsilon}(\xi, \tau):=\exp \left(\frac{1}{\varepsilon} \int_{\tau}^{\xi}\left[\lambda_{2}\left(u_{\varepsilon}(\eta)\right)-\eta\right] d \eta-\frac{1}{2 \varepsilon}\left(\tau-\xi_{\varepsilon}\right)^{2}\right) \text {. }
$$

Therefore, $\left|b_{\varepsilon}(\xi)\right| \leq\left(C_{2} / \varepsilon^{3}\right)\left|\xi-\eta_{\varepsilon}\right| \max \left\{F_{\varepsilon}(\xi, \tau): \tau \in I\left[\xi, \eta_{\varepsilon}\right]\right\}$, where $I\left[\xi, \eta_{\varepsilon}\right]$ denotes the closed interval with extremes $\xi$ and $\eta_{\varepsilon}$.

If $\tau_{0}$ is a critical point of $F_{\varepsilon}(\xi, \cdot)$ for each fixed $\xi$, then we can check that

$$
\frac{d^{2}}{d \tau^{2}} F_{\varepsilon}\left(\xi, \tau_{0}\right)=-\frac{1}{\varepsilon} \dot{\lambda}_{2}\left(u_{\varepsilon}\left(\tau_{0}\right)\right) F_{\varepsilon}\left(\xi, \tau_{0}\right)>0
$$

to conclude that $F_{\varepsilon}(\xi, \cdot)$ cannot have a local maximum point.

Therefore, $\max \left\{F_{\varepsilon}(\xi, \tau): \tau \in I\left[\xi, \eta_{\varepsilon}\right]\right\}=\max \left\{F_{\varepsilon}\left(\xi, \eta_{\varepsilon}\right), F_{\varepsilon}(\xi, \xi)\right\} \leq E_{\varepsilon}(\xi)$ and so

$$
\left|b_{\varepsilon}(\xi)\right| \leq \frac{C_{2}}{\varepsilon^{3}}\left|\xi-\eta_{\varepsilon}\right| E_{\varepsilon}(\xi) .
$$

From (5.6), (5.8), and (5.9) we obtain (5.5) with $C=\max \left\{C_{1}, C_{2}\right\}$.

The next result is an immediate consequence of Proposition 5.3. We omit the proof.

Corollary 5.4. There exists a constant $C$ that is independent of $\varepsilon$ such that

$$
\begin{gathered}
\left|\dot{v}_{\varepsilon}(\xi)\right| \leq \frac{C}{\varepsilon^{3}} \exp \left(-\frac{1}{2 \varepsilon}\left(\xi_{\varepsilon}-\xi\right)^{2}\right)\left[1+\xi_{\varepsilon}-\xi\right] ; \quad \xi \leq \xi_{\varepsilon}, \\
\left|\dot{v}_{\varepsilon}(\xi)\right| \leq \frac{C}{\varepsilon^{3}} ; \quad \xi_{\varepsilon} \leq \xi \leq \eta_{\varepsilon}, \\
\left|\dot{v}_{\varepsilon}(\xi)\right| \leq \frac{C}{\varepsilon^{3}} \exp \left(-\frac{1}{2 \varepsilon}\left(\xi-\eta_{\varepsilon}\right)^{2}\right)\left[1+\xi-\eta_{\varepsilon}\right] ; \quad \eta_{\varepsilon} \leq \xi .
\end{gathered}
$$

In the following, $s_{r}=\max \left\{s, \lambda_{2}\left(u_{r}\right)\right\}$. 
Corollary 5.5. For any $0<\alpha<L$ we have

(a) $\dot{v}_{\varepsilon} \rightarrow 0$ uniformly on $[s-L, s-\alpha] \cup\left[s_{r}+\alpha, s_{r}+L\right]$;

(b) if $s<\lambda_{2}\left(u_{r}\right)$ then $\dot{v}_{\varepsilon} \rightarrow 0$ uniformly on $\left[s-\alpha, \lambda_{2}\left(u_{r}\right)+\alpha\right]$ (here $2 \alpha<\lambda_{2}\left(u_{r}\right)-s$ );

(c) $v_{\varepsilon} \rightarrow v_{s}$ uniformly on $(-\infty, s-\alpha] \cup\left[s_{r}+\alpha,+\infty\right)$.

Proof. Since $\xi_{\varepsilon} \rightarrow s$ and $\eta_{\varepsilon} \rightarrow s_{r}$ (Proposition 5.2) we conclude from Corollary 5.4 that there exists a constant $C$ and $\varepsilon_{0}>0$ such that

$$
\left|\dot{v}_{\varepsilon}(\xi)\right| \leq \frac{C}{\varepsilon^{3}} \exp \left(-\frac{\alpha^{2}}{8 \varepsilon}\right) ; \quad \varepsilon<\varepsilon_{0},
$$

if $\xi \in[s-L, s-\alpha] \cup\left[s_{r}+\alpha, s_{r}+L\right]$ (in this case $C$ depends only on $\alpha$ and $L$ ) or if $\xi \in\left[s+\alpha, \lambda_{2}\left(u_{r}\right)-\alpha\right]$ when $s<\lambda_{2}\left(u_{r}\right)=s_{r}$ (in this case $C$ depends only on $\alpha$ ).

The conclusions of (a) and (b) follow immediately by the inequality above when $\varepsilon \rightarrow$ $0^{+}$.

Now if $\eta<\xi \leq s-\alpha$, then from (5.10) we have

$$
\left|v_{\varepsilon}(\eta)-v_{\varepsilon}(\xi)\right| \leq \int_{\eta}^{\xi}\left|\dot{v}_{\varepsilon}(\theta)\right| d \theta \leq C\left[I_{\varepsilon}^{1}(\xi, \eta)+I_{\varepsilon}^{2}(\xi, \eta)\right]
$$

where

$$
\begin{aligned}
I_{\varepsilon}^{1}(\xi, \eta) & =\frac{1}{\varepsilon^{3}} \int_{\eta}^{\xi} \exp \left(-\frac{1}{2 \varepsilon}\left(\xi_{\varepsilon}-\theta\right)^{2}\right) d \theta \\
I_{\varepsilon}^{2}(\xi, \eta) & =\frac{1}{\varepsilon^{3}} \int_{\eta}^{\xi}\left(\xi_{\varepsilon}-\theta\right) \exp \left(-\frac{1}{2 \varepsilon}\left(\xi_{\varepsilon}-\theta\right)^{2}\right) d \theta \\
& =\frac{1}{\varepsilon^{2}} \exp \left(-\frac{1}{2 \varepsilon}\left(\xi_{\varepsilon}-\xi\right)^{2}\right)-\frac{1}{\varepsilon^{2}} \exp \left(-\frac{1}{2 \varepsilon}\left(\xi_{\varepsilon}-\eta\right)^{2}\right),
\end{aligned}
$$

and $C$ is a constant that does not depend on $\xi, \eta$, and $\varepsilon$.

Taking $\sigma=\frac{\xi_{\varepsilon}-\theta}{\sqrt{2 \varepsilon}}$ and $\eta \rightarrow-\infty$ we obtain

$$
I_{\varepsilon}^{1}(\xi,-\infty)=\frac{\sqrt{2 \varepsilon}}{\varepsilon^{3}} \int_{\frac{\xi_{\varepsilon}-\xi}{\sqrt{2 \varepsilon}}}^{+\infty} \exp \left(-\sigma^{2}\right) d \sigma
$$

Since $\xi_{\varepsilon} \rightarrow s$ there exists $\varepsilon_{0}>0$ such that $\xi_{\varepsilon}-\xi>\frac{\alpha}{2}$ if $\varepsilon<\varepsilon_{0}$. Consequently, for $\varepsilon<\varepsilon_{0}$ we have $I_{\varepsilon}^{1}(\xi,-\infty) \leq \frac{\sqrt{2}}{\varepsilon^{2} \sqrt{\varepsilon}} \int_{\frac{r}{2 \sqrt{2 \varepsilon}}}^{+\infty} \exp \left(-\sigma^{2}\right) d \sigma$ and

$$
I_{\varepsilon}^{2}(\xi,-\infty)=\frac{1}{\varepsilon^{2}} \exp \left(-\frac{1}{2 \varepsilon}\left(\xi_{\varepsilon}-\xi\right)^{2}\right) \leq \frac{1}{\varepsilon^{2}} \exp \left(-\frac{\alpha^{2}}{8 \varepsilon}\right) .
$$

Making $\eta \rightarrow-\infty$ in $(5.12)$ we have

$$
\left|v_{l}-v_{\varepsilon}(\xi)\right| \leq \frac{\sqrt{2}}{\varepsilon^{2} \sqrt{\varepsilon}} \int_{\frac{\alpha}{2 \sqrt{2 \varepsilon}}}^{+\infty} \exp \left(-\sigma^{2}\right) d \sigma+\frac{1}{\varepsilon^{2}} \exp \left(-\frac{\alpha^{2}}{8 \varepsilon}\right) ; \quad \varepsilon<\varepsilon_{0} .
$$

Hence we obtain (c) on $(-\infty, s-\alpha]$ since

$$
\lim _{\varepsilon \rightarrow 0} \frac{1}{\varepsilon^{2} \sqrt{\varepsilon}} \int_{\frac{\alpha}{2 \sqrt{2 \varepsilon}}}^{+\infty} \exp \left(-\sigma^{2}\right) d \sigma=0=\lim _{\varepsilon \rightarrow 0} \frac{1}{\varepsilon^{2}} \exp \left(-\frac{\alpha^{2}}{8 \varepsilon}\right) .
$$

Similarly we prove (c) on $\left[s_{r}+\alpha,+\infty\right)$. 
Now we can prove the weak convergence of the viscosity wavefans to the delta-shock $S_{\delta}$

Theorem 5.6. Suppose $u_{l}>u_{r}$ and $\lambda_{2}\left(u_{r}\right) \leq s$. Then $\left(u_{\varepsilon}, v_{\varepsilon}\right) \rightarrow S_{\delta}$ as $\varepsilon \rightarrow 0^{+}$.

Proof. As a consequence of Corollary 5.5 (item (c)) and from Lemma 5.1 (item (b)) we have the uniform convergence $\left(u_{\varepsilon}, v_{\varepsilon}\right) \rightarrow\left(u_{s}, v_{s}\right)$ on each closed interval contained on $(-\infty, s) \cup(s,+\infty)$, since $s_{r}=\lambda_{2}\left(u_{r}\right)$. Putting together this fact with (ii) from Theorem 4.4 of Sec. 4 we can apply Theorem 2.4 of Sec. 2 to obtain immediately the result.

Next we give a sketch of the proof of the weak convergence $\left(u_{\varepsilon}, v_{\varepsilon}\right) \rightarrow\left(u_{s}, v_{s}\right)$ when $u_{l}>u_{r}$ and $s<\lambda_{2}\left(u_{r}\right)$.

First, we observe that the family $\left\{v_{\varepsilon}\right\}$ is uniformly bounded on each closed interval $I \subset$ $\left(s, \lambda_{2}\left(u_{r}\right)\right)$. In fact, denoting by $|I|$ the length of $I$ and writing $\left|v_{\varepsilon}\left(\xi_{0}\right)\right|=\frac{1}{|I|} \int_{I} \mid v_{\varepsilon}\left(\xi_{0}\right)-$ $v_{\varepsilon}(\eta)+v_{\varepsilon}(\eta) \mid d \eta$ we have, for each $\xi_{0} \in I$ :

$$
\left|v_{\varepsilon}\left(\xi_{0}\right)\right| \leq \frac{1}{|I|}\left(\left\|\dot{v}_{\varepsilon}\right\|_{L^{\infty}(I)}\right) \int_{I}\left|\xi_{0}-\eta\right| d \eta+\frac{1}{|I|} \int_{I}\left|v_{\varepsilon}(\eta)\right| d \eta \leq C_{1}+\frac{K_{I}}{|I|},
$$

where $K_{I}$ and $C_{I}$ are constants that depend only $I . K_{I}$ is obtained from (4.21) and $C_{I}$ is such that $|I|\left\|\dot{v}_{\varepsilon}\right\|_{L^{\infty}(I)} \leq C_{I}$ since $\dot{v}_{\varepsilon} \rightarrow 0$ uniformly on $I$ (Corollary 5.5 item (b)).

Second, in view of the boundedness above and the uniform convergence $\dot{v}_{\varepsilon} \rightarrow 0$ on closed intervals contained in $\left(s, \lambda_{2}\left(u_{r}\right)\right)$ we can prove that there exists a constant $v_{m}$ such that $v_{\varepsilon} \rightarrow v_{m}$ uniformly on $\left[\lambda_{2}\left(u_{r}\right)-\alpha, \lambda_{2}\left(u_{r}\right)+\alpha\right]$ for each $\alpha>0$. So, from Lemma 5.1 and Corollary 5.5 (item (c)) we can conclude that $\left(u_{\varepsilon}, v_{\varepsilon}\right) \rightarrow\left(u_{s}, v\right)$ uniformly on each closed interval contained in $(-\infty, s) \cup\left(s, \lambda_{2}\left(u_{r}\right)\right) \cup\left(\lambda_{2}\left(u_{r}\right),+\infty\right)$ where

$$
v(\xi)= \begin{cases}v_{l} ; & \xi<s, \\ v_{m} ; & s<\xi<\lambda_{2}\left(u_{r}\right), \\ v_{r} ; & \lambda_{2}\left(u_{r}\right)<\xi .\end{cases}
$$

Third, we apply Theorem 2.4 to conclude that $\left(u_{\varepsilon}, v_{\varepsilon}\right) \rightarrow\left(u_{s}, v+c \delta_{s}\right)$ where

$$
c=s\left[v_{m}-v_{l}\right]-\left[v_{m} \lambda_{2}\left(u_{r}\right)-v_{l} \lambda_{2}\left(u_{l}\right)\right] .
$$

The constant that could appear multiplying $\delta_{\lambda_{2}\left(u_{r}\right)}$ is trivially zero.

At last, we prove that $c=0$, which is equivalent to proving that $\left(u_{r}, v_{m}\right)$ belongs to the 1 -shock curve $S_{1}\left(u_{l}, v_{l}\right)$ if $v_{l} \neq 0$. In view of the linearity of the second equation in (4.1) we need only to prove that $c=0$ for the following two cases.

Case 1: $0<v_{l}$ and $v_{r}=\bar{v}_{l}+1$ where $\bar{v}_{l}$ is such that

$$
s\left[\bar{v}_{l}-v_{l}\right]=\lambda_{2}\left(u_{r}\right) \bar{v}_{l}-\lambda_{2}\left(u_{l}\right) v_{l},
$$

i.e., the state $\left(u_{r}, \bar{v}_{l}\right)$ belongs to the 1 -shock curve $S_{1}\left(u_{l}, v_{l}\right)$. We can verify easily that $\bar{v}_{l}>v_{l}$.

In this case we claim that $v_{\varepsilon}$ is uniformly bounded on an interval that contains $s$. In fact, if $v_{\varepsilon}$ is not monotonic then, since $v_{r}>\bar{v}_{l}>v_{l}>0$ there exists a unique maximum point $\tau_{\varepsilon}$ of $v_{\varepsilon}$ and a unique point $\alpha_{\varepsilon}$ satisfying $v_{l}<v_{\varepsilon}(\xi)<v_{\varepsilon}\left(\alpha_{\varepsilon}\right)=v_{r}$ if $\xi \in\left(-\infty, \alpha_{\varepsilon}\right)$ and $v_{\varepsilon}(\eta)>v_{r}$ if $\eta \in\left(\alpha_{\varepsilon}, \infty\right)$. Therefore, from Theorem 2.3 we obtain

$$
0<\int_{-\infty}^{\alpha_{\varepsilon}}\left[v_{\varepsilon}(\xi)-v_{l}\right] d \xi+\int_{\alpha_{\varepsilon}}^{+\infty}\left[v_{\varepsilon}(\xi)-v_{r}\right] d \xi=\alpha_{\varepsilon}\left[v_{r}-v_{l}\right]-\left[v_{r} \lambda_{2}\left(u_{r}\right)-v_{l} \lambda_{2}\left(u_{l}\right)\right] .
$$


So, from (5.14) we have

$$
\alpha_{\varepsilon}\left[v_{r}-v_{l}\right]>\left[v_{r} \cdot \lambda_{2}\left(u_{r}\right)-v_{1} \lambda_{2}\left(u_{l}\right)\right]=s\left[v_{r}-v_{l}\right]+\lambda_{2}\left(u_{r}\right)-s,
$$

i.e., $\alpha_{\varepsilon}-s>k:=\frac{\lambda_{2}\left(u_{r}\right)-s}{r_{r}-v_{l}}$. We observe that the positive constant $k$ depends only on $u_{l}, u_{r}, v_{l}$, and $v_{r}$.

Thus, $v_{l}<v_{\xi}(\xi)<v_{r} ; \xi \in(-\infty, s+k]$. since $(-\infty, s+k] \subset\left(-\infty, a_{\varepsilon}\right)$. The last inequalities remain valid if $v_{\varepsilon}$ is monotonic.

Hence, $\lim _{\varepsilon \rightarrow 0} \int_{s-3}^{s+3} v_{\varepsilon}(\xi) d \xi=\beta\left[v_{m}+v_{l}\right]$. where we have applied the Dominated Convergence Theorem.

On the other hand, integrating the second equation in (4.1) on the set $[s-\beta, s) \cup$ $(s, s+\beta] \subset[-\infty, s+k]$ and taking $\varepsilon \rightarrow 0$ we find

$$
\lim _{\varepsilon \rightarrow 0} \int_{s-\beta}^{s+3} v_{\xi} d \xi=\lim _{\varepsilon \rightarrow 0}\left[\varepsilon \dot{v}_{\xi}(\xi)+\xi v_{\varepsilon}(\xi)-v_{\Xi}(\xi) g\left(u_{\varepsilon}(\xi)\right)\right]_{s-3}^{s+3}=c+\beta\left[v_{m}+v_{l}\right] .
$$

Therefore $c=0$ and $v_{\varepsilon}$ converges weakly to $v$ in (5.13) with $v_{m}=v_{l} \frac{s-\lambda_{2}\left(u_{1}\right)}{s-\lambda_{2}\left(u_{r_{r}}\right)}$.

Case 2: $v_{l}=0<v_{r}$. We prove that $c=0$ for this case using the same arguments as in Case 1 above. So, $v_{\S}$ converges weakly to $v$ in (5.13) with $v_{m}=0=v_{l}$.

Now, for the general case we prove weak convergence by using Cases 1 and 2 above and the linearity of (4.1) with respect to $v$. For example, if $v_{r} \leq 0<v_{l}$, then the (unique) solution of $(4.1)-(4.2)$ is $\left(u_{\varepsilon}, v_{\varepsilon}^{1}-v_{\xi}^{2},-v_{\varepsilon}^{3}\right)$ where $\left(u_{\varepsilon}, v_{\varepsilon}^{1}\right),\left(u_{\varepsilon}, v_{\xi}^{2}\right)$, and $\left(u_{\varepsilon}, v_{\varepsilon}^{3}\right)$ are the solutions of (4.1) satisfying $u_{\Sigma}(-\infty)=u_{l}, u_{\S}(+\infty)=u_{r}, v_{\xi}^{1}(-\infty)=v_{l}, v_{\varepsilon}^{1}(+\infty)=\bar{v}_{l}+1$, $v_{\Sigma}^{2}(-\infty)=0, v_{\varepsilon}^{2}(+\infty)=\bar{v}_{l}+1, v_{\varepsilon}^{3}(-\infty)=0$, and $v_{\varepsilon}^{3}(+\infty)=-v_{r}$. We observe that $\left(u_{\varepsilon}, v_{\varepsilon}^{1}\right)$ corresponds to Case 1 and that $\left(u_{\xi}, v_{\xi}^{2}\right)$ and $\left(u_{\xi}, v_{\xi}^{3}\right)$ correspond to Case 2 .

So, in this example we obtain $\left(u_{\varepsilon}, v_{\varepsilon}^{1}-v_{\varepsilon}^{2},-v_{\varepsilon}^{3}\right) \rightarrow\left(u_{s}, v\right)$ where $v$ is the function in (5.13) with $v_{m}=v_{l} \frac{s-\lambda_{2}\left(u_{1}\right)}{s-\lambda_{2}\left(u_{r}\right)}$.

Acknowledgment. This work is based on my Doctorate thesis at the University of Campinas (UNICAMP), Brazil. I would like to thank my advisor Milton Lopes for his admirable manner of advising me always with encouragements, even after I have finished my thesis.

\section{REFERENCES}

[1] C. M. Dafermos, Solution of the Riemann problem for a class of hyperbolic systems of conservation laws by the viscosity method, Arch. Rat. Mech. Anal. 52, 1-9 (1973)

[2] C. M. Dafermos and R. J. DiPerna, The Riemann problem for certain classes of hyperbolic systems of conservation laws, J. Differential Equations 20, 90-114 (1976)

[3] H. T. Fan, A limiting viscosity approach to the Riemann problem for materials exhibiting change of phase II, Arch. Rat. Mech. Anal. 116, 317-338 (1992)

[4] H. T. Fan, One-phase Riemann problem and wave interactions in systems of conservation laws of mixed type, SIAM J. Math. Anal. (4) 24, 840-865 (1993)

[5] J. Hu, A limiting viscosity approach to Riemann solutions containing delta-shock waves for nonstrictly hyperbolic conservation laws, Quart. Appl. Math. (2) 55, 361 373 (1997)

[6] F. Huang, Existence and uniqueness of discontinuous solutions for a hyperbolic system, Proc. Roy. Soc. Edinburgh 127, 11931205 (1997)

[7] B. L. Keyfitz and H. C. Kranzer, A viscosity approximation to a system of conservation laws with no classical Riemann solution, Nonlinear Hyperbolic Problems, Lecture Notes in Math., Vol. 1402, Springer-Verlag, NY, 1989, pp. 185197 
[8] B. L. Keyfitz and H. C. Kranzer, A strictly hyperbolic system of conservation laws admitting singular shocks, in Nonlinear Evolution Equations that Change Type, IMA Vol. Math. Appl. 27, 107-125 (1990)

[9] D. J. Korchinski, Solution of a Riemann problem for a $2 \times 2$ system of conservation laws possessing no classical weak solution, Ph.D. Thesis, Adelphi University, 1977

[10] M. Slemrod, A limiting viscosity approach to the Riemann problem for materials exhibiting change of phase, Arch. Rat. Mech. Anal. 105, 327-365 (1989)

[11] M. Slemrod and A. E. Tzavaras, A limiting viscosity approach for the Riemann problem in isentropic gas dynamics, Indiana Univ. Math. J. (4) 38, 1047-1074 (1989)

[12] D. Tan, Riemann problem for hyperbolic systems of conservation laws with no classical wave solutions, Quart. Appl. Math. (4) 51, 765-776 (1993)

[13] D. Tan, T. Zhang, and Y. Zheng, Delta-shock waves as limits of vanishing viscosity for hyperbolic systems of conservation laws, J. Differential Equations 112, 1-32 (1994)

[14] A. E. Tzavaras, Elastic as limit of viscoelastic response, in a context of self-similar viscous limits, J. Differential Equations 123, 305-341 (1995)

[15] A. E. Tzavaras, Wave interactions and variation estimates for self-similar zero-viscosity limits in systems of conservation laws, Arch. Rational Mech. Anal. 135, 1-60 (1996) 